\title{
Study on the correlation among dysbacteriosis, imbalance of cytokine and the formation of intrauterine adhesion
}

\author{
Xingping Zhao ${ }^{1}$, Qun Zhao $^{1}$, Xiuting Zhu ${ }^{1}$, Huan Huang ${ }^{1}$, Xing Wan $^{2}$, Rui Guo ${ }^{2}$, Yanqiu Zhao ${ }^{2}$, Dan Chen ${ }^{2}$, \\ Dabao Xu ${ }^{1}$ \\ ${ }^{1}$ Department of Gynecology, Third Xiangya Hospital of Central South University, Changsha 410013, China; ${ }^{2}$ Scientific Research Centre, The Third \\ Hospital Affiliated to the Chinese University of Hong Kong, Shenzhen 518172, China \\ Contributions: (I) Conception and design: D Xu, D Chen; (II) Administrative support: None; (III) Provision of study materials or patients: D Xu; (IV) \\ Collection and assembly of data: X Zhao; (V) Data analysis and interpretation: X Zhao; (VI) Manuscript writing: All authors; (VII) Final approval of \\ manuscript: All authors. \\ Correspondence to: Dabao Xu, MD, PhD. Department of Gynecology, Third Xiangya Hospital of Central South University, 138 Tongzipo Rd., \\ Changsha 410013, China. Email: dabaoxu@yahoo.com; Dan Chen, PhD. The Third Hospital Affiliated to the Chinese University of Hong Kong \\ Shenzhen, No. 53 Ai Xin Road, Longgang District, Shenzhen 518172, China. Email: 1183243412@qq.com.
}

Background: Intrauterine adhesion (IUA) is one of the most important causes of female infertility, while iatrogenic endometrial injury is the main, but not the entire, cause of IUA. The microorganisms of the female reproductive tract play an important role in its health and disease. The imbalance of immune regulation caused by the imbalance of reproductive tract dysbacteriosis may be an important link in the formation mechanism of uterine cavity adhesion.

Methods: We prospectively enrolled 30 patients diagnosed with IUA and 30 women with a history of intrauterine surgery, but without IUA, as control subjects. All participants were diagnosed with hysteroscopy while two swabs-one being leucorrhea drawn from the middle of the vagina and the other being cervical mucus drawn from the cervical canal-were taken. The bacterial load and community were identified by $16 \mathrm{~S}$ rDNA quantitative polymerase chain reaction and pyrosequencing. Immunocytokines in serum were quantitatively detected by human T-helper cytokine kit. The correlation between Th cytokines and microorganisms in IUA and non-IUA groups was analyzed.

Results: Compared with non-IUA participants at the phylum level, patients with IUA had a significantly higher percentage of firmicutes in most samples, while the diversity of bacteria was significantly decreased. Some species that were members of vaginal and cervical canal bacterial phyla, including Euryarchaeota, Acidobacteria, Chlamydiae, Chlorobi, Planctomycetes and TM6 (Dependentiae), almost disappeared. The quantity in serum of IUA patients of classical proinflammatory cytokines IL-6 and TNF- $\gamma$, released from immune cells, also known as profibrotic cytokines, were significantly higher than that of the non-IUA women in our study $(\mathrm{P}<0.05)$

Conclusions: IUA is characterized by an increased bacterial burden, decreased diversity of bacterial communities in the vagina/cervical canal, and increased immune cytokines of pro-fibrosis, which may predict new and more effective therapeutic schemes for the treatment of IUA.

Keywords: Intrauterine adhesion (IUA); vaginal microbe; cytokines

Submitted Aug 30, 2019. Accepted for publication Oct 31, 2019.

doi: $10.21037 /$ atm.2019.11.124

View this article at: http://dx.doi.org/10.21037/atm.2019.11.124 


\section{Introduction}

Intrauterine adhesion (IUA) was first reported by Joseph Asherman, and is thus also known as Asherman syndrome $(1,2)$. Most patients with IUAs have fibrous band adhesions formed in the anterior and posterior walls of the uterus, and these vascular fibrous tissue adhesion bands are accompanied by different amounts of white blood cell infiltration (3). Adhesions begin to form when fibers and inflammatory exudate appear on the surface of the damaged endometrium, and then gradually develop into denser fibrous bands, eventually forming mass adhesions. Adhesion can lead to partial or total uterine cavity obliteration which reduces the implantation area of embryos. IUA hinders the endometrial blood supply and reduces the endometrial receptivity which is not conducive to the growth of the embryos $(4,5)$. The common clinical manifestations of IUA include hypomenorrhea, amenorrhea, recurrent abortion, and secondary infertility. There are various causes of IUAs (6), and its prevalence rate increases yearly, which is mainly due to the increased incidence of iatrogenic endometrial trauma such as artificial abortion and postpartum curettage. Other causes include genital tuberculosis, pelvic irradiation, and uterine surgery (including myomectomy and surgery for uterine malformation, etc.) (7). Hooker et al. (8) reported that curettage was the most important reason for the formation of IUA during pregnancy. Iatrogenic endometrial trauma is the main cause of IUA, but this alone cannot fully explain the mechanism of adhesion formation. It is necessary to explore the potential pathogenic factors of IUA, so as to formulate specific preventive measures for IUA based on the study of these factors.

Here, we investigated the role of the uterine microenvironment: the microbiome in the formation mechanism of IUA. It has been known for some time that the microbiome of the female reproductive tract plays an important role both in maintaining a healthy state and in the development of diseases in the female reproductive system. It is also widely acknowledged that lactic acid-producing microorganisms dominate the vaginal microbial community (9), maintaining a relatively healthy reproductive tract microenvironment by lowering the vaginal $\mathrm{PH}$ value. Previous studies have shown that vaginal dysbacteriosis will lead to a variety of diseases, such as postpartum endometritis, preterm delivery, pelvic inflammatory disease, spontaneous abortion, delivery of low-birth-weight babies, etc. $(10-12)$.
The etiology and progression mechanism of IUA caused by microorganisms is similar to that of other damaged tissues and organs with fibrosis (13). Tissue damage and bacterial imbalance not only promote inflammation, but also release a large number of proinflammatory and profibrotic cytokines by recruiting and activating innate and adaptive immune systems. For example, when infected by bacteria, Th1 response results in a release of large amounts of proinflammatory cytokines for the clearance of pathogens. The damaged tissues need an immune response, mainly of a profibrotic nature that is conducive to tissue repair. Meanwhile, a set of cytokines, including IL-25, IL-33, TSLP il-25, il-33, and TSLP are released by damaged epithelial cells, while other cells can directly or indirectly promote the development of $\mathrm{Th} 2 \mathrm{immune}$ response that promotes fibrosis (14). In previous studies, many researchers have discussed the specific immune pathways and immune cytokines that lead to fibrosis. However, few studies have directly investigated the relationship between the microbes in the uterine environment and the immune response induced by these microbes in the formation of IUAs. As far as we know, the pathological changes of IUA also affect the physiology and metabolites of the uterus, further affecting the diversity of vaginal microorganisms. Therefore, we investigated the association and interaction among IUA and microbes in the vagina. In this study, the data of 30 IUA patients and 30 healthy women were collected to analyze the correlation among vaginal microorganisms, immune cytokines, and the formation of IUAs.

\section{Methods}

The trial enrolled 30 women aged between 20 and 40 years who were newly diagnosed with IUA during hysteroscopy examination at the Third Xiangya Hospital of Central South University between January $1^{\text {st }}$, 2018, and May $31^{\text {st }}, 2018$, IUAs were scored by one surgeon applying the American Fertility Society (AFS) classification system (15) (IUA group). IUAs were scored as follows: 1-4 (mild), 5-8 (moderate), and 9-12 (severe). Thirty women aged between 20 and 40 years who took routine gynecological health examination including hysteroscopy in the same hospital during the same period were recruited as the control group (non-IUA group). None of the participants had used vaginal medications, received cervical treatment, or performed douching within the previous 7 days, nor had they engaged in sexual activity within the previous 2 days. All participants had a history of uterine cavity operation, and none were 
Table 1 Demographic and clinical characteristics of the study groups

\begin{tabular}{lrcc}
\hline Basic clinical characteristics & VD $(\mathrm{n}=30)$ & $\mathrm{CD}(\mathrm{n}=30)$ & $\mathrm{P}$ value \\
\hline Age (year), mean $\pm \mathrm{SD}$ & $30.90 \pm 4.31$ & $31.6 \pm 4.42$ & 0.561 \\
Height $(\mathrm{m})$, mean $\pm \mathrm{SD}$ & $1.59 \pm 0.04$ & $1.58 \pm 0.06$ & 0.526 \\
Weight $(\mathrm{kg})$, mean $\pm \mathrm{SD}$ & $54.78 \pm 7.12$ & $53.37 \pm 6.29$ & 0.418 \\
$\mathrm{BMl}\left(\mathrm{kg} / \mathrm{m}^{2}\right)$, mean $\pm \mathrm{SD}$ & $21.60 \pm 2.46$ & $21.30 \pm 2.19$ & 0.639 \\
IUD, $\mathrm{n}(\%)$ & $0(0)$ & $0(0)$ & $\mathrm{NE}$ \\
$\begin{array}{lcc}\text { Other gynecological disease, } \\
\mathrm{n}(\%)\end{array}$ & $0(0)$ & $0(0)$ & $\mathrm{NE}$ \\
Endocrine disorders, $\mathrm{n}(\%)$ & $0(0)$ & $0(0)$ & $\mathrm{NE}$ \\
\hline
\end{tabular}

SD, standard deviation; BMI, body mass index; NE, not estimable (due to nullity of category in both groups); IUD, intrauterine device.

diagnosed with endocrine or autoimmune disorders, cancer, severe pelvic adhesion, hysteromyoma, endometriosis, adenomyosis, or acute inflammation. All participants were subjected to two swabs by a doctor: one of leucorrhea drawn from the middle of the vagina (marked zhong) and one of cervical mucus drawn from the cervical canal (marked kou) in the follicular phase of the menstrual cycle. All swabs were used to collect vaginal specimens which were immediately stored at $-80{ }^{\circ} \mathrm{C}$ for DNA extraction, while anticoagulant blood vessels were centrifuged to separate the serum for cytokine detection.

DNA was extracted from 120 samples by using Fast DNA $^{\circledR}$ SPIN Kit for Soil (MP Biomedicals) according to manufacturer's protocols, and the concentration and quality of purified DNA was determined via a spectrophotometer at $230 \mathrm{~nm}$ (A230) and $260 \mathrm{~nm}$ (A260; NanoDrop). Subsequently, the V3 and V4 region of the bacterial $16 \mathrm{~S}$ rDNA gene was amplified by PCR using the following primers: 341FCCTACGGGNBGCASCAG/805RGACTA CNVGGGTATCTAATCC. PCR reactions were performed in a $25 \mu \mathrm{L}$ mixture containing $5 \mu \mathrm{L}$ of $5 \times$ GC Buffer, $0.5 \mu \mathrm{L}$ of KAPA dNTP Mix, $0.5 \mu \mathrm{L}$ of KAPA HiFi HotStart DNA Polymerase, $0.5 \mu \mathrm{L}$ of each primer $(10 \mathrm{pM})$, and $50-100 \mathrm{ng}$ of template DNA. PCR reaction cycling included $95^{\circ} \mathrm{C}$ for $3 \mathrm{~min}$, followed by 25 cycles at $95^{\circ} \mathrm{C}$ for $30 \mathrm{~s}, 55^{\circ} \mathrm{C}$ for $30 \mathrm{~s}$, and $72{ }^{\circ} \mathrm{C}$ for $30 \mathrm{~s}$, and a final extension at $72{ }^{\circ} \mathrm{C}$ for 5 min. PCR clean up used AMPure XP beads to purify the $16 \mathrm{~S} \mathrm{~V} 3$ and V4 amplicon away from the free primers and primer dimer species. Purified product was amplified by PCR using the following primers: 341F CCTACGGGNBGCASCAG/805R GACTACNVGGGTATCTAATCC, where the barcode was an eight-base sequence unique to each sample. PCR reactions were performed in $25 \mu \mathrm{L}$ mixture containing $5 \mu \mathrm{L}$ of $5 \times$ GC Buffer, $0.75 \mu \mathrm{L}$ of KAPA dNTP Mix, $0.5 \mu \mathrm{L}$ of KA-PA HiFi HotStart DNA Polymerase, $1.5 \mu \mathrm{L}$ of each primer $(10 \mathrm{pM})$, and $5 \mu \mathrm{L}$ of purified product. PCR reaction cycling included $95^{\circ} \mathrm{C}$ for $3 \mathrm{~min}$, followed by 8 cycles at $95{ }^{\circ} \mathrm{C}$ for $30 \mathrm{~s}, 55^{\circ} \mathrm{C}$ for $30 \mathrm{~s}$, and $72{ }^{\circ} \mathrm{C}$ for $30 \mathrm{~s}$, and a final extension at $72{ }^{\circ} \mathrm{C}$ for $5 \mathrm{~min}$. The amplicons were subsequently purified by AMPure XP beads to clean up the final library before quantification. Last, purified amplicons were pooled in equimolar and pairedend sequenced $(2 \times 250)$ on an Illumina MiSeq platform according to the standard protocols.

Fast Length Adjustment of SHort reads (FLASH) was used to merge paired-end reads from next-generation sequencing (1). Low-quality reads were filtered by fastq quality_filter (-p 90 -q 25 -Q33) in FASTX Toolkit 0.0 .14 , and chimera reads were removed by USEARCH 64 bit v8.0.1517. The number of reads for each sample was normalized based on the smallest size of samples by random subtraction. OTUs were aligned by the UCLUST algorithm with a $97 \%$ identity, and taxonomically classified using the SILVA 16S rRNA database v128. Alpha and beta diversities were generated in the Quantitative Insights Into Microbial Ecology (QIIME) and calculated based on weighted and unweighted Unifrac distance matrices.

Th1/Th2 cytokines in serum samples were quantified by Legendplex Assay (Human T helper Cytokine Panel, BioLegend). The correlation between Th cytokines and microorganisms was analyzed.

\section{Results}

\section{Basic clinical characteristics of participants}

Between January $1^{\text {st }}, 2018$, and May $31^{\text {st }}, 2018$, 60 women were recruited to either the IUA group (30 patients with IUA) or the non-IUA group (30 healthy women). Baseline characteristics between the two groups including age, height, weight, BMI, IUD, and other gynecological diseases and endocrine disorders, showed no statistical differences (Table 1). Two groups of participants had a 1- or 2-time history of induced abortion, AFS scores were moderate to severe in the IUA group patients (11 moderate IUA, 19 severe IUA), and no adhesions were diagnosed in the non-IUA group. Each participant was subjected to a vaginal 
swab (marked zhong) and a cervical canal swab (marked kou); a total of 120 samples were available for highthroughput sequencing.

\section{Data quality control and optimization}

Samples were distinguished according to barcode sequences, and the extracted data were stored in the standard fastq files. Data quality evaluation of sequencing samples was conducted according to the fastq file. The number of paired reads in the sample, the base ratio with $Q$ value greater than 20 , the mass value in the sample higher than 30, and the GC content of the sample data are listed in Table 2.

\section{Operational taxonomic unit (OTU) clustering and species notes}

As indicated by the $97 \%$ sequence similarity level, Uclust method in the QIIME software package was used for OTU clustering analysis. The classification results of all the samples at the levels of phylum, class, order, family, and genus were analyzed. Based on the cumulative histogram of species in the top 15 of abundance, the microorganism composition differences among samples were compared and presented in a list. The community structure analysis results of the whole sample at the phylum and genus level are shown in Figure 1. At the phylum level, Firmicutes, Actinobacteria, Proteobacteria Bacteroidetes, and Fusobacteria constituted five of the most predominant phyla in the following regions: the cervical canal of the IUA group $(69.55 \%, 11.05 \%, 6.85 \%, 8.40 \%$, and $1.53 \%$, respectively), the middle of the vagina of the IUA group (69.20\%, $11.16 \%, 6.84 \%, 8.38 \%$ and $1.53 \%$, respectively), the cervical canal of the non-IUA group (41.67\%, $14.03 \%$, $14.10 \%, 11.90 \%$, and $8.61 \%$, respectively), and the middle of the vagina of the non-IUA group $(41.68 \%, 14.41 \%$, $14.03 \%, 11.77 \%$, and $8.61 \%$, respectively). In the IUA group, the proportion of Firmicutes in most samples was more than $60 \%$, while the diversity of bacteria was significantly reduced, with, for example, the Euryarchaeota, Acidobacteria, Chlamydiae, Chlorobi, Planctomycetes, and TM 6 (Dependentiae) phyla almost all disappearing. Statistical analysis also showed that the proportion of Firmicutes in IUA group was significantly higher than that in non-IUA group, and there were significant differences in Lactobacillus, Cetobacterium and Bifidobacterium $(\mathrm{P}<0.05)$ (Figure 2).
Table 2 Sequencing sample data output and quality evaluation statistics

\begin{tabular}{|c|c|c|c|c|}
\hline Sample & PE_reads & Q20 (\%) & Q30 (\%) & GC (\%) \\
\hline \multicolumn{5}{|l|}{ Sample (VD) } \\
\hline A101kou & 7702 & 97.66 & 95.89 & 51.99 \\
\hline A101zhong & 137219 & 97.73 & 96.03 & 52.48 \\
\hline A102kou & 129313 & 97.88 & 96.25 & 54.06 \\
\hline A102zhong & 158034 & 97.87 & 96.20 & 53.62 \\
\hline A103kou & 147018 & 97.90 & 96.33 & 53.48 \\
\hline A103zhong & 137450 & 97.78 & 96.12 & 53.55 \\
\hline A104kou & 144249 & 97.33 & 95.71 & 53.24 \\
\hline A104zhong & 164513 & 97.69 & 96.09 & 53.66 \\
\hline A105kou & 165721 & 96.78 & 95.12 & 52.15 \\
\hline A105zhong & 122235 & 97.71 & 96.09 & 51.85 \\
\hline A55kou & 135442 & 97.56 & 95.90 & 51.66 \\
\hline A55zhong & 118568 & 97.77 & 96.08 & 50.83 \\
\hline A56kou & 159992 & 96.29 & 94.62 & 51.86 \\
\hline A56zhong & 149826 & 97.66 & 96.00 & 52.42 \\
\hline A57kou & 138006 & 97.81 & 96.25 & 51.85 \\
\hline A57zhong & 139832 & 97.76 & 96.15 & 52.04 \\
\hline A59kou & 147775 & 97.85 & 96.25 & 52.06 \\
\hline A59zhong & 135591 & 97.71 & 96.09 & 51.97 \\
\hline A62kou & 144575 & 97.75 & 96.15 & 52.25 \\
\hline A62zhong & 124962 & 97.88 & 96.26 & 51.82 \\
\hline A63kou & 148910 & 97.85 & 96.28 & 51.97 \\
\hline A63zhong & 127930 & 96.90 & 95.26 & 51.82 \\
\hline A65kou & 140301 & 97.43 & 95.86 & 51.85 \\
\hline A65zhong & 136975 & 97.88 & 96.29 & 52.00 \\
\hline A67kou & 190662 & 97.38 & 95.84 & 52.56 \\
\hline A67zhong & 180826 & 93.56 & 91.86 & 51.88 \\
\hline A68kou & 146383 & 97.73 & 96.17 & 52.39 \\
\hline A68zhong & 137618 & 97.87 & 96.30 & 52.06 \\
\hline A74kou & 162131 & 97.02 & 95.37 & 52.45 \\
\hline A74zhong & 139848 & 97.48 & 95.84 & 52.19 \\
\hline A75kou & 219948 & 90.84 & 88.99 & 51.31 \\
\hline A75zhong & 151254 & 97.83 & 96.22 & 52.13 \\
\hline A77kou & 163859 & 97.35 & 95.79 & 51.97 \\
\hline A77zhong & 149796 & 97.64 & 96.09 & 52.25 \\
\hline A78kou & 153324 & 97.76 & 96.15 & 52.23 \\
\hline A78zhong & 146487 & 97.81 & 96.18 & 52.01 \\
\hline A82kou & 145602 & 97.74 & 96.15 & 52.06 \\
\hline A82zhong & 166547 & 97.01 & 95.36 & 52.14 \\
\hline A84kou & 152770 & 97.56 & 95.94 & 52.31 \\
\hline
\end{tabular}

Table 2 (continued) 
Table 2 (continued)

\begin{tabular}{|c|c|c|c|c|}
\hline Sample & PE_reads & Q20 (\%) & Q30 (\%) & GC (\%) \\
\hline A84zhong & 153188 & 98.01 & 96.46 & 52.19 \\
\hline A85kou & 151276 & 97.99 & 96.48 & 52.16 \\
\hline A85zhong & 150678 & 97.68 & 96.06 & 52.35 \\
\hline A86kou & 168598 & 97.40 & 95.81 & 52.35 \\
\hline A86zhong & 178303 & 97.71 & 96.09 & 51.81 \\
\hline A88kou & 163560 & 97.82 & 96.21 & 53.23 \\
\hline A88zhong & 142423 & 97.77 & 96.13 & 53.22 \\
\hline A89kou & 142954 & 97.30 & 95.68 & 52.13 \\
\hline A89zhong & 150183 & 97.80 & 96.15 & 51.65 \\
\hline A90kou & 133372 & 97.63 & 95.97 & 52.20 \\
\hline A90zhong & 179171 & 97.77 & 96.11 & 52.52 \\
\hline A92kou & 170181 & 97.34 & 95.74 & 52.44 \\
\hline A92zhong & 160550 & 97.84 & 96.19 & 52.04 \\
\hline A95kou & 89529 & 97.33 & 95.71 & 53.15 \\
\hline A95zhong & 137312 & 96.80 & 95.14 & 51.98 \\
\hline A96kou & 170898 & 97.51 & 95.90 & 52.19 \\
\hline A96zhong & 147055 & 97.93 & 96.36 & 51.92 \\
\hline A97kou & 145110 & 97.80 & 96.15 & 52.36 \\
\hline A97zhong & 134741 & 97.87 & 96.19 & 52.65 \\
\hline A98kou & 176908 & 97.61 & 96.05 & 52.71 \\
\hline A98zhong & 138250 & 97.86 & 96.23 & 52.57 \\
\hline \multicolumn{5}{|l|}{ Sample (CD) } \\
\hline B45kou & 164485 & 91.62 & 89.74 & 50.67 \\
\hline B45zhong & 106021 & 97.15 & 95.44 & 52.14 \\
\hline B46kou & 158701 & 95.22 & 93.53 & 51.15 \\
\hline B46zhong & 152103 & 96.23 & 94.57 & 52.07 \\
\hline B47kou & 196685 & 94.39 & 92.70 & 52.07 \\
\hline B47zhong & 184698 & 91.86 & 90.05 & 51.79 \\
\hline B48kou & 175716 & 91.76 & 89.95 & 51.50 \\
\hline B48zhong & 137551 & 92.64 & 90.93 & 52.22 \\
\hline B50kou & 142384 & 94.40 & 92.65 & 51.91 \\
\hline B50zhong & 139164 & 93.14 & 91.36 & 51.38 \\
\hline B51kou & 144403 & 96.19 & 94.63 & 49.57 \\
\hline B51zhong & 121438 & 97.86 & 96.29 & 49.11 \\
\hline B53kou & 174179 & 84.30 & 81.95 & 50.05 \\
\hline B53zhong & 122428 & 93.59 & 91.86 & 51.52 \\
\hline B54kou & 182883 & 94.45 & 92.76 & 51.91 \\
\hline B54zhong & 158752 & 92.72 & 90.99 & 51.09 \\
\hline B55kou & 165246 & 96.89 & 95.26 & 53.00 \\
\hline B55zhong & 133782 & 97.77 & 96.20 & 53.35 \\
\hline B58kou & 140464 & 94.06 & 92.32 & 52.29 \\
\hline
\end{tabular}

Table 2 (continued)
Table 2 (continued)

\begin{tabular}{|c|c|c|c|c|}
\hline Sample & PE_reads & Q20 (\%) & Q30 (\%) & GC (\%) \\
\hline B58zhong & 159405 & 96.00 & 94.37 & 53.23 \\
\hline B59kou & 172022 & 95.80 & 94.15 & 52.16 \\
\hline B59zhong & 139824 & 95.67 & 94.03 & 51.87 \\
\hline B60kou & 158457 & 93.91 & 92.25 & 51.89 \\
\hline B60zhong & 136713 & 95.88 & 94.31 & 51.70 \\
\hline B62kou & 143449 & 93.70 & 91.95 & 51.94 \\
\hline B62zhong & 165476 & 94.18 & 92.43 & 51.86 \\
\hline B63kou & 135867 & 95.00 & 93.34 & 51.52 \\
\hline B63zhong & 158737 & 96.24 & 94.60 & 51.03 \\
\hline B64kou & 174318 & 95.00 & 93.27 & 52.07 \\
\hline B64zhong & 142343 & 95.13 & 93.43 & 52.01 \\
\hline B65kou & 185074 & 90.67 & 88.80 & 51.14 \\
\hline B65zhong & 146121 & 93.47 & 91.63 & 51.90 \\
\hline B66kou & 174426 & 93.47 & 91.75 & 51.75 \\
\hline B66zhong & 203522 & 92.99 & 91.22 & 51.39 \\
\hline B67kou & 181635 & 92.17 & 90.38 & 51.42 \\
\hline B67zhong & 122453 & 97.03 & 95.43 & 52.32 \\
\hline B68kou & 141852 & 94.35 & 92.65 & 51.83 \\
\hline B68zhong & 139997 & 94.76 & 93.07 & 51.80 \\
\hline B69kou & 144565 & 87.20 & 85.12 & 51.02 \\
\hline B69zhong & 232848 & 86.72 & 84.62 & 50.57 \\
\hline B70kou & 101082 & 96.37 & 94.77 & 52.70 \\
\hline B70zhong & 162161 & 97.73 & 96.12 & 52.92 \\
\hline B71kou & 150398 & 91.81 & 90.01 & 51.31 \\
\hline B71zhong & 123454 & 95.50 & 93.78 & 51.09 \\
\hline B72kou & 161306 & 96.11 & 94.47 & 51.73 \\
\hline B72zhong & 153212 & 96.97 & 95.37 & 51.75 \\
\hline B73kou & 188604 & 91.29 & 89.43 & 51.17 \\
\hline B73zhong & 165029 & 84.44 & 82.28 & 50.15 \\
\hline B74kou & 179825 & 96.50 & 94.99 & 52.05 \\
\hline B74zhong & 326549 & 83.51 & 81.04 & 49.50 \\
\hline B75kou & 128424 & 96.36 & 94.75 & 52.17 \\
\hline B75zhong & 168641 & 96.71 & 95.06 & 52.20 \\
\hline B76kou & 188426 & 89.99 & 88.11 & 51.40 \\
\hline B76zhong & 131517 & 97.40 & 95.77 & 52.65 \\
\hline B77kou & 174054 & 88.58 & 86.67 & 50.70 \\
\hline B77zhong & 108945 & 92.95 & 91.21 & 51.22 \\
\hline B78kou & 164436 & 93.73 & 91.99 & 51.43 \\
\hline B78zhong & 141726 & 95.54 & 93.62 & 51.84 \\
\hline B79kou & 186324 & 90.85 & 88.99 & 51.55 \\
\hline B79zhong & 166931 & 88.20 & 86.19 & 50.97 \\
\hline
\end{tabular}




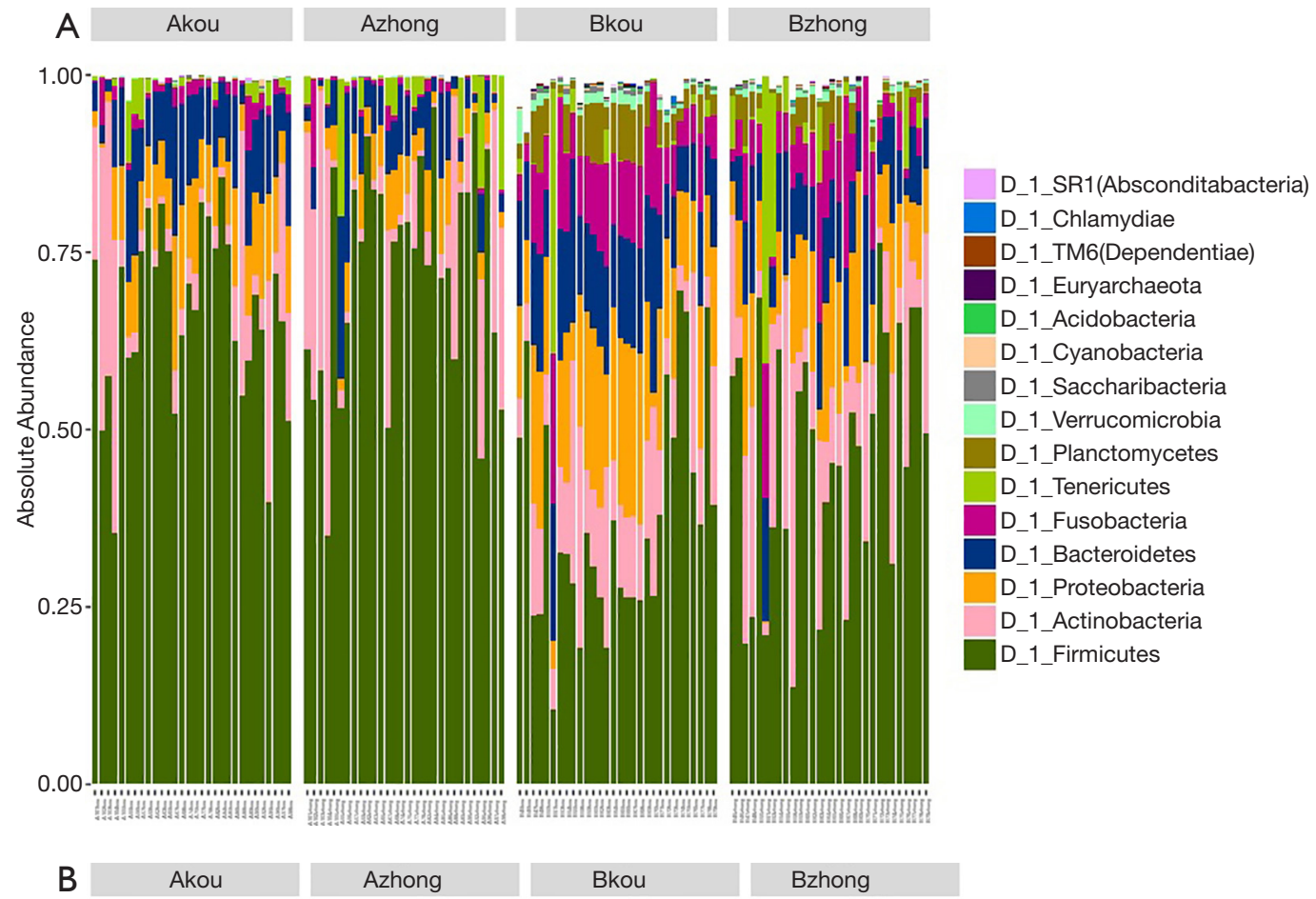

$1.00-$

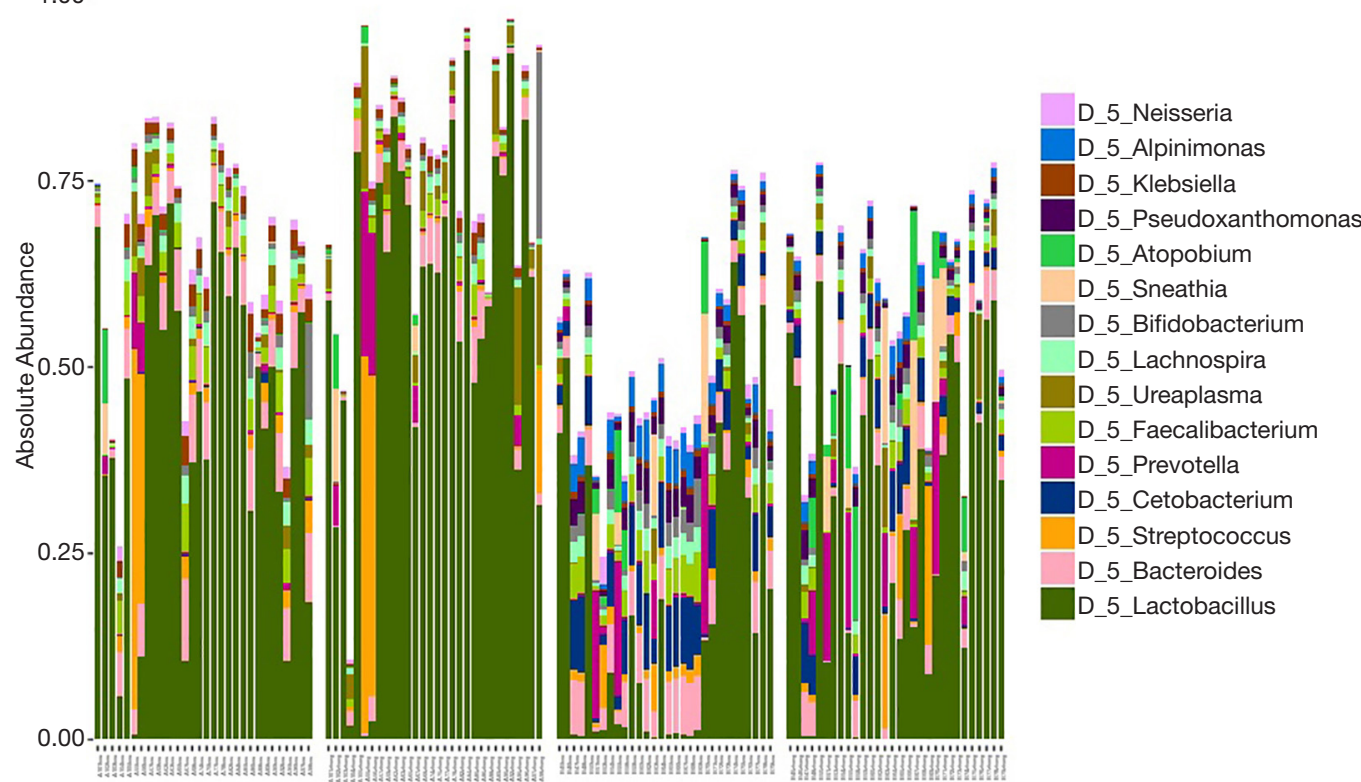

Figure 1 The cumulative histogram of species in the top 15 of abundance at the phylum (A) and genus (B) level.

\section{Alpha diversity analysis}

The Alpha diversity index includes the species abundance (chao1, ACE), the diversity index of integrating species abundance and uniformity (Shannon, Simpson), and the observed species, which reflects the low OTU coverage of samples in Good's coverage and OTU number. Among them, abundance measures species in a single sample by estimating the OTU number, while the diversity index measures the heterogeneity of communities. As shown in Figure 3, the species diversity in the middle vagina and cervix of the IUA group was significantly lower than that of 
A
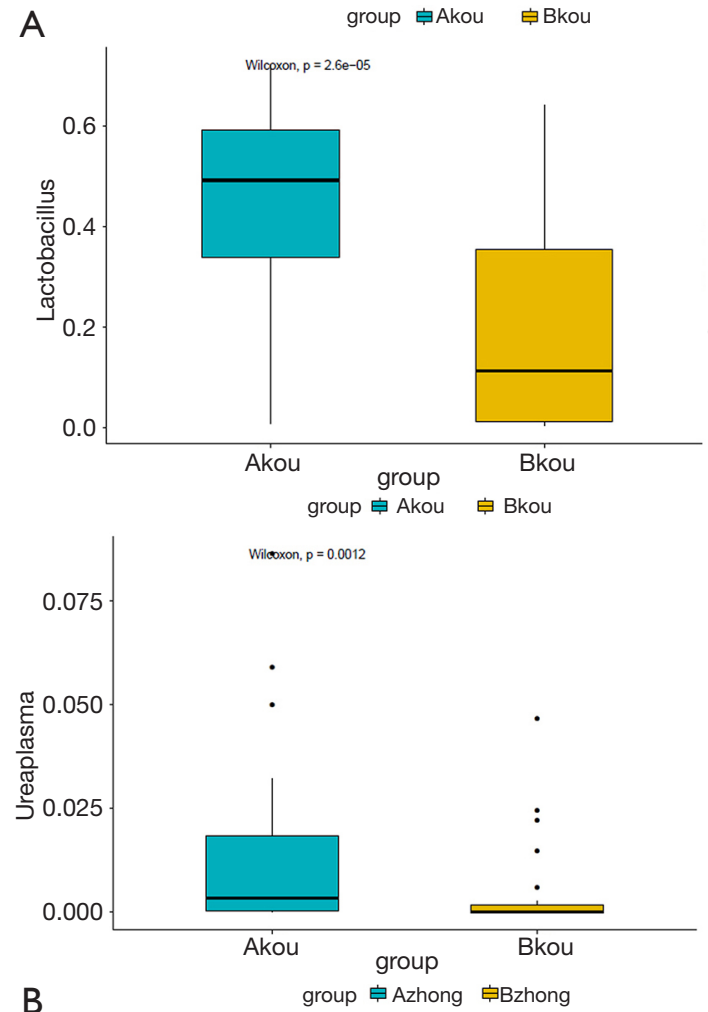

B
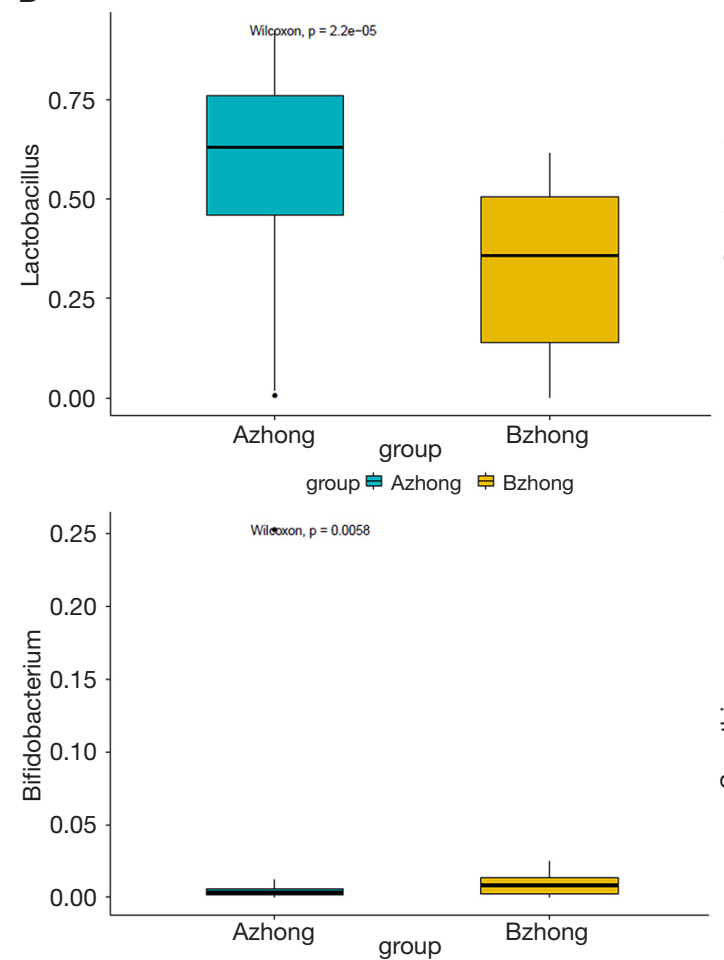
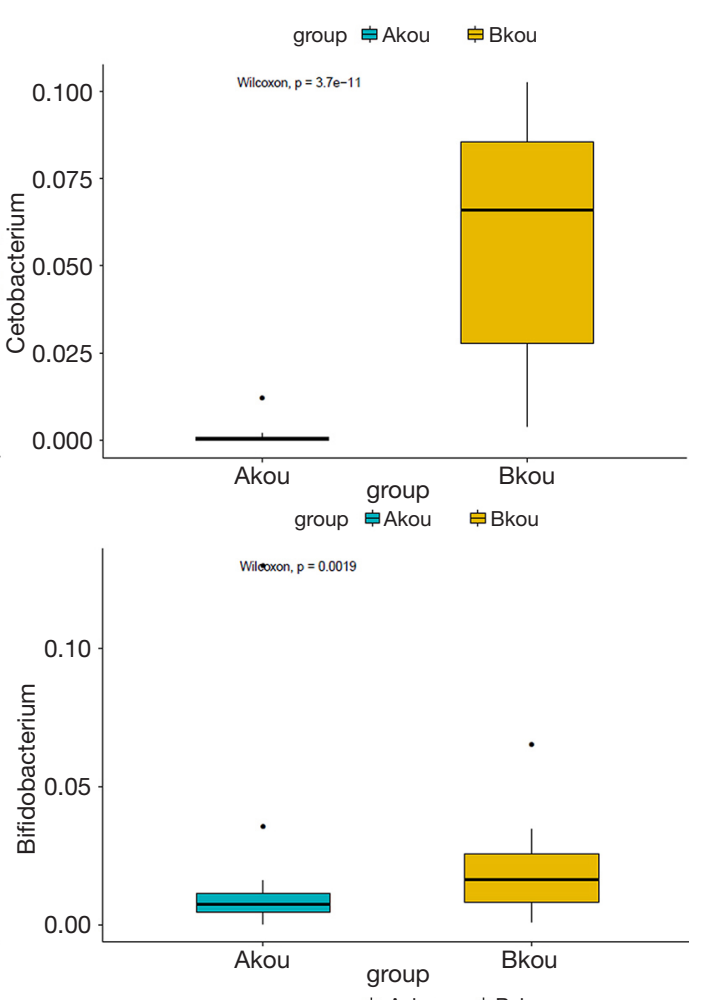

group 追 Azhong 追Bzhong
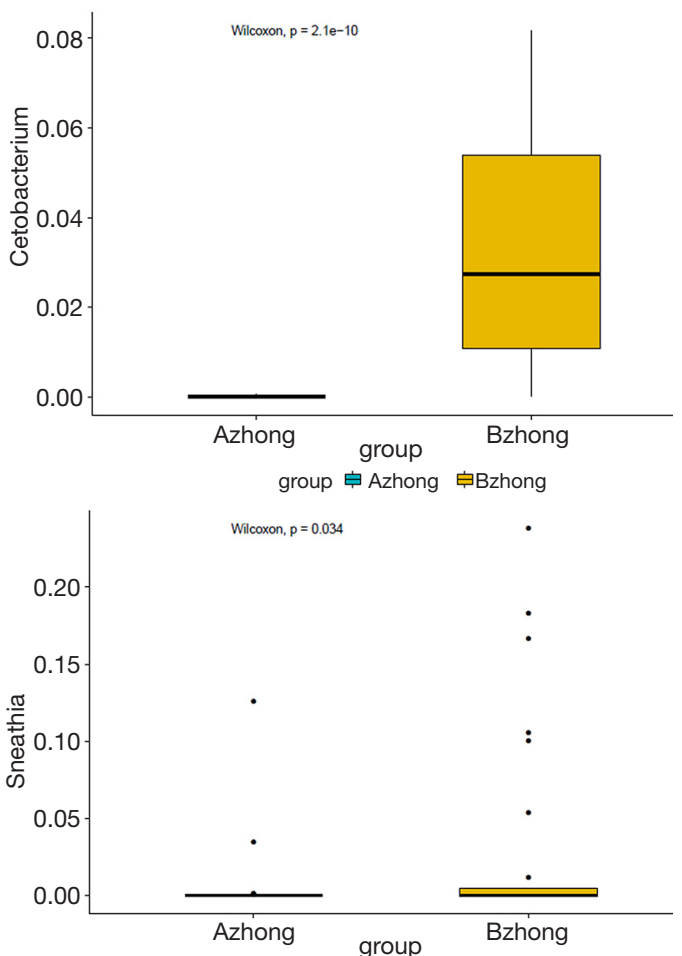

Figure 2 Significant differences of species between the IUA and non-IUA groups in the vagina (A) and cervical canal (B) at the phylum level. IUA, intrauterine adhesion. 

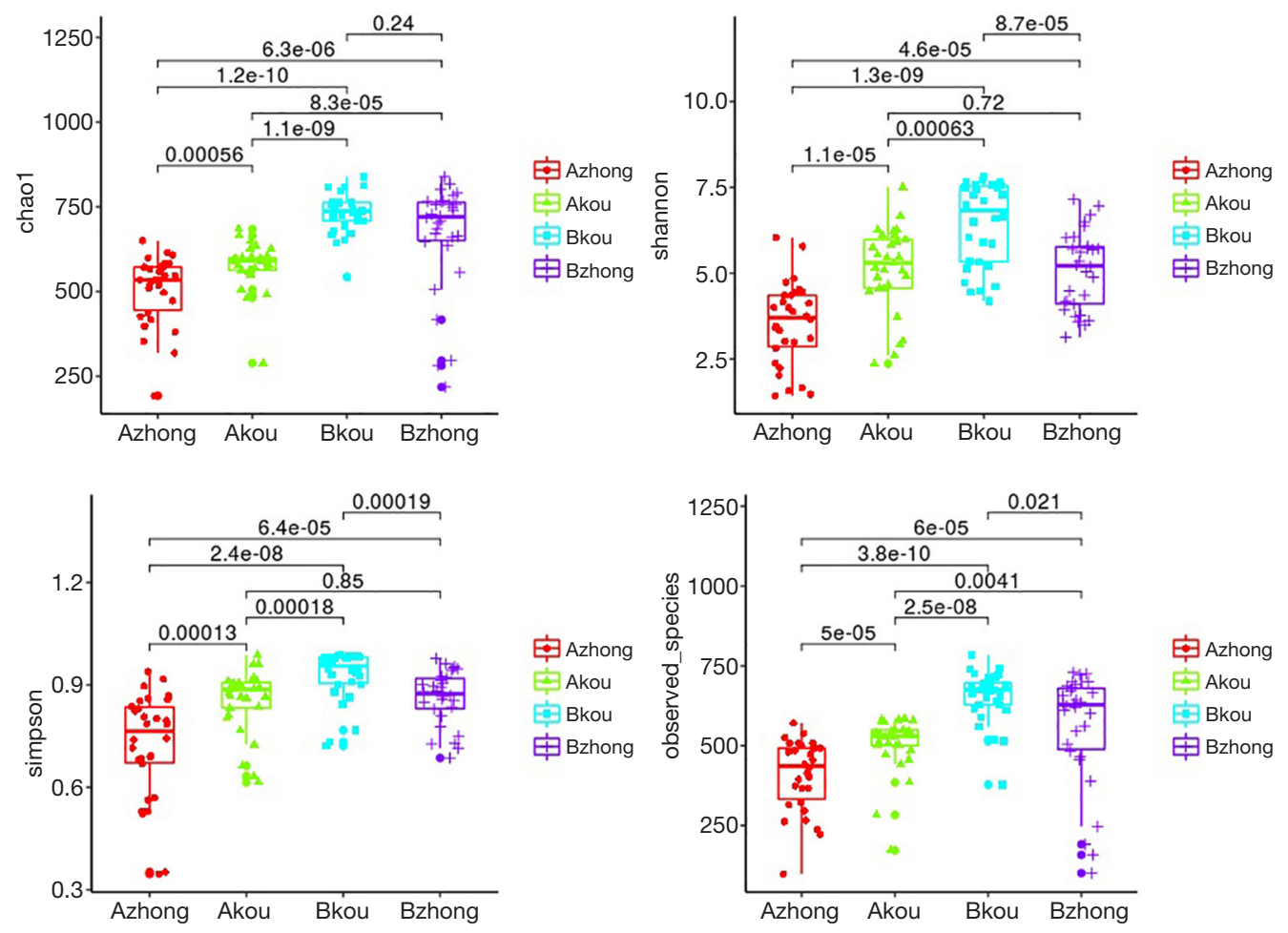

Figure 3 Alpha diversity analysis in the middle vagina and cervix of the IUA group was significantly different from that of the non-IUA group. IUA, intrauterine adhesion.

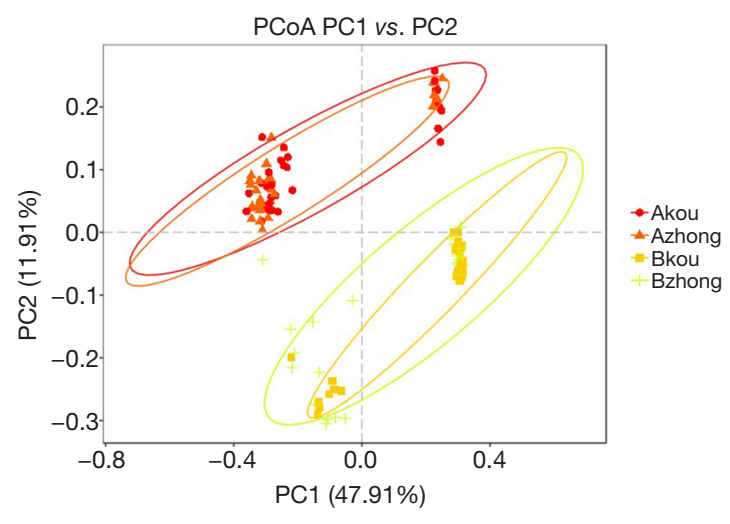

Figure 4 PCoA of Beta diversity analysis for the abundance and composition of species in the vagina and cervix between the groups.

the non-IUA group, and the species diversity in the middle vagina of the IUA group was the lowest $(\mathrm{P}<0.001)$.

\section{Beta diversity analysis}

The principal coordinate analysis $(\mathrm{PCoA})$ was based on the distance matrix to find the principal coordinates. Through a series of eigenvalues and eigenvectors following sorting, the top several main characteristic values were selected to effectively to find the "main" elements and structure between the two groups of sample data visualization of similarities or differences (Figure 4). The abundance and composition of species in the vagina and cervix were more similar in the same group, but the difference between the groups was significant.

\section{Differences of cytokines in the IUA and non-IUA groups}

The cytokine kit quantitatively detected 13 human cytokines secreted by Th1/Th2 immune cells, including IL-2, 4, 5, 6, 9, 10, 13, 17A, 17F, 21, 22, along with IFN- $\gamma$, and TNF- $\alpha$. IL-6 and IFN- $\gamma$ were significantly different between the two groups, with the IUA group being significantly higher than the non-IUA group $(\mathrm{P}<0.05)$. Correlation analysis was conducted between immune cytokines and microbial genera, and the correlation heat map is shown in Figure 5. At the genus level, Aerophilus, Brevundimonas, Ezakiella, Mobiluncus, Peptoniphilus, and Prevotella 6 were highly 

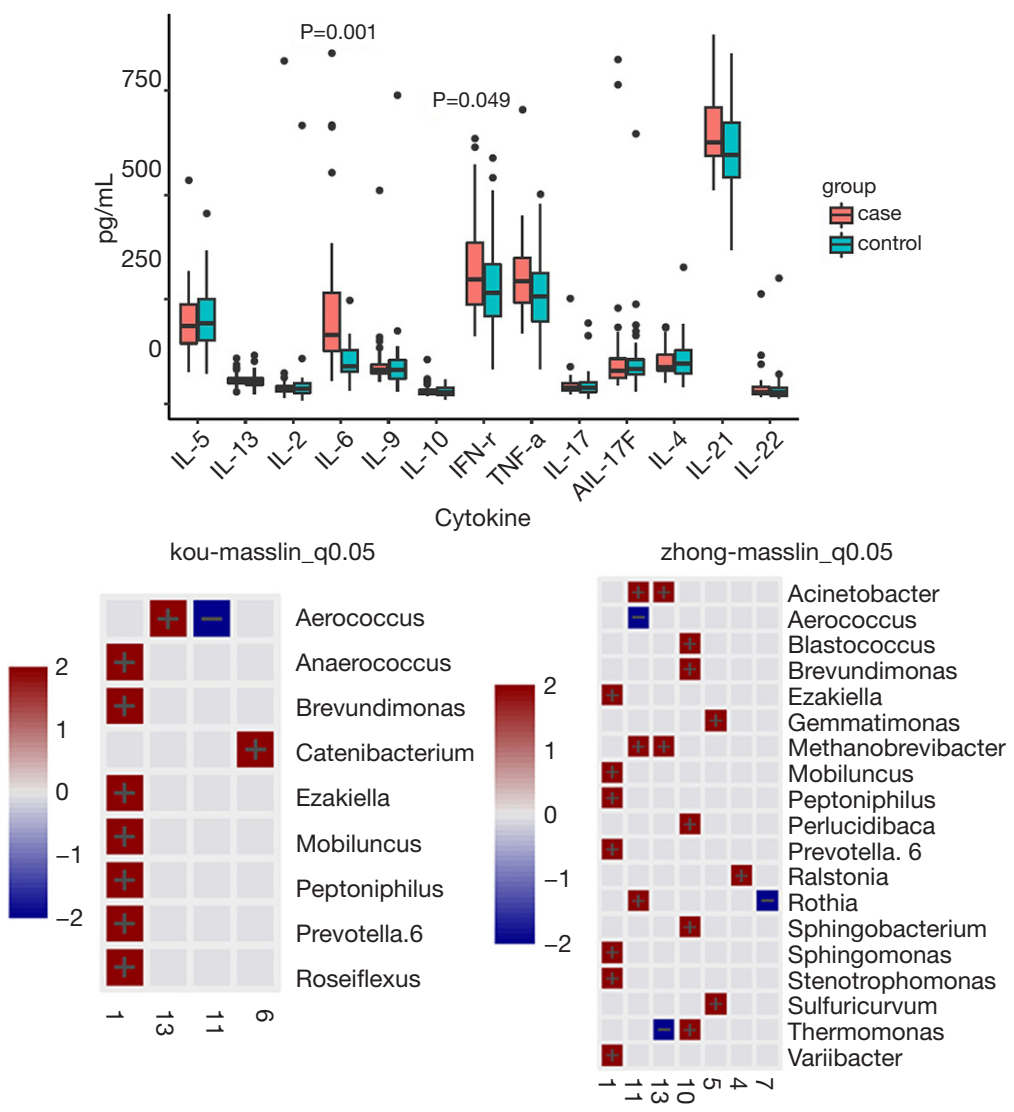

Figure 5 Correlation heat map between immune cytokines and microbial genus level.

correlated with cytokines.

\section{Discussion}

With the wide application of hysteroscopy in clinical practice, the incidence and diagnosis rate of IUA in females of childbearing age has sharply increased to $2.2-36.8 \%$ $(16,17)$. Iatrogenic endometrial trauma is the primary condition of IUA, which is mainly caused by curettage during pregnancy. It has been reported that more than $90 \%$ of IUAs are caused by curettage $(18,19)$. Other causes include removal of pregnancy residues, cesarean section, diagnostic curettage, myomectomy, and correction of uterine anomalies. Taskin $e t$ al. reported that IUA could be formed after hysteroscopic myomectomy, with a probability of $31.3 \%$ (20). If multiple myomectomy was performed, the incidence of IUA would rise to $45.5 \%$, and $6.7 \%$ after hysteroscopic resection of a septum. Although hysteroscopic adhesiolysis (HA) has been widely used in clinic (21), the high recurrence rate of IUA and poor pregnancy outcomes after HA present a great challenge for clinical treatment and prevention (22).

To date, researchers have studied the role of microorganisms in the lower reproductive tract in reproductive system tumors, vaginitis, adverse pregnancy, and other diseases (23-29); however, the interaction between vaginal microbiota, immune response, and IUA has not been investigated.

Microorganisms are the richest source of biodiversity on earth. The investigation of microbial diversity has important theoretical and practical significance for human diseases, environmental governance, and the exploitation and utilization of microbial resources. The humancoexisting microorganisms have important influences on human growth and development, physiology and pathology, immunity, nutrition metabolism, and other processes (30-35). Most of these microbiotas have a mutually beneficial relationship with their human hosts, and only a few opportunistic pathogens can cause chronic infections or other serious diseases under certain conditions. These 
microbial communities are also considered the body's first line of defense against foreign pathogens, competing to eliminate other invasive pathogens. As is well known, the symbiotic microorganisms are very important to the human body, but incredibly, people know little about the community composition and function of the microorganisms. Furthermore, we barely understand how microorganisms interact with each other, or how they can form a balanceable ecoenvironment. At present, many investigators are focusing on the research of human microorganisms, in order to get a better idea of the real role of these microorganisms in human health and disease (36).

There are many kinds of microorganisms in the vagina, and 29 kinds of microorganisms have been isolated from vaginal secretions (37-39). The most important one is lactobacillus $(40,41)$, and the other microflora present in the vagina are mainly composed of bacteria, fungi, protozoa, and virus. Under normal circumstances, the microbes in the vagina can reach an equilibrium state. Any factors that cause vaginal microecological imbalance can lead to inflammation, such as long-term application of antibiotics, frequent intercourse, vaginal flush, and a decline in estrogen (42). The balance of vaginal microecology can effectively resist the invasion of various foreign pathogens. The female reproductive tract is highly susceptible to bacterial infection. Once the immunity of the body is reduced, foreign bacteria or parasitic bacteria in the body may increase infection, causing cervicitis, endometritis, etc. (43). The main cause of IUA is injury to the endometrium associated with reproductive tract infection. Patients with IUA are usually infected with a variety of reproductive tract pathogens. Once these pathogens invade the vagina, they can enter the uterine cavity through the cervix, causing endometritis, salpingitis, or pelvic inflammatory disease. Abortion preoperative cervicitis or endometritis in the case of no cure pre-abortion can lead bacteria into the uterine cavity and then cause intrauterine infection or increase the chances of IUA formation. In this study, compared with the control group, patients with IUA had a higher bacterial load and density, but less diversity in the vagina/ cervix, a condition which may cause persistent damage by pathogens in the impairment of the endometrium. Some of the bacterial communities in IUA patients that disappeared in our study may play an important role in maintaining the health of the reproductive tract, and their absence may lead to a compromised repair function of the endometrium. After the iatrogenic injury of the endometrium occurred, the load of the opportunistic bacteria increased and the essential bacteria became absent, so that the normal repair function of endometrium tissue was reduced and replaced by fiber repair which lost normal function.

To our knowledge, tissue injury and inflammation are important causes of tissue fibrosis. This study focused on the pathways from tissue damage and inflammation to fibrosis. The vaginal immune function is a component of the systemic immune function, including humoral and cellular immunity. The functions of systemic and local immunity jointly form an immune response network. When the local mucosa is attacked by pathogens, the immune response will quickly be generated in the blood and other mucosal sites. Some immune mediators released by immune $\mathrm{T}$ cells have both profibrotic and antifibrotic properties. After iatrogenic damage to the endometrium, pathological biological bacteria of the vagina and cervical canal invade into the endometrium, causing local inflammation and triggering the immune response of the endometrium, which will eliminate some pathogenic microorganisms and further change the composition of bacteria. Endometrial injury destroys the function of the surface barrier and increases the entry of harmless symbiotic bacteria or their products into the endometrium, resulting in the aggravation of the inflammatory response and fibrosis repair of the endometrium. Immune Th cells can be divided into two subgroups according to different functions, TH1 and TH2 cells, which play an important role in Th response. For inflammatory diseases, most available literature shows that Th1 expression is weak, while Th2 expression is strong, leading to the imbalance of TH1 with TH2. Cytokines secreted by immune Th cells are key regulators of immune responses to health and disease. As mentioned above, damaged endometrial epithelial cells release a series of immune cytokines, which directly or indirectly promote the development of Th2 immune response that is thought to promote fibrosis (44). The innate immune cells can release classical proinflammatory cytokines IL-6, IL-1, and TNF- $\gamma$ which are also profibrotic. In our study, the IL- 6 and IFN- $\gamma$ quantities in the serum of IUA patients were significantly higher than those of the non-IUA women $(\mathrm{P}<0.05)$. In repeated peritonitis models, inflammatory Th1 cells and cytokines IL-6 and IFN- $\gamma$ are essential factors in the occurrence and development of fibrosis (45). It was found that cytokine IL-6 increased the Th1 cell survival rate and up-regulated IFN- $\gamma$ production, while negative feedback decreased MMP activity. Therefore, the fibrogenic effect of IL-6 is mainly due to the reduced degradation of matrix protein. Other studies have explained the role of IL-6 
in promoting fibrosis by regulating the TGF- $\beta$ pathway $(46,47)$. Profibrotic effects of IL-6 were observed in fibrosis models of different organs, including the lung $(48,49)$, heart $(50,51)$, kidney (47), and eye (52).

Microorganisms play a role in mediating inflammation/ immune response to trigger related pathways, a phenomenon which has become an area of intense research. IUAs seriously endanger the reproductive health of childbearingage females, and its pathogenesis is complex. Therefore, it is important to study the pathogenesis of IUAs and to find effective treatment. We analyzed the differences of vaginal microbiological structure between patients with IUA and healthy women. Our study found that vaginal microbial imbalance can cause endometrial inflammation and inflammation factors or related cytokines released in great quantities. Proinflammatory cytokines IL-6 and IFN- $\gamma$ were massively increased in IUA patients and played a role in promoting fibrosis, which in turn caused fibrinogen accumulation, extracellular matrix deposition, and eventually induced IUAs. A comprehensive understanding of microorganisms and immune function will allow us to better clarify the role of microorganisms and immune function in the development of IUA. From a new perspective of vaginal dysbacteriosis mediating a Th1-Th2 imbalance, the mechanism of IUA was revealed. Moreover, it was found that fibrosis could be successfully prevented by inhibiting pathogens, inflammatory factors, and cytokines. These findings provide a new platform for the prevention and treatment of IUA targeted at microbial and cytokine intervention.

\section{Acknowledgments}

Funding: This study is supported by National Key Research and Development Program of China (Grant No.2018YFC1004800), Natural Science Foundation of China (Grant No. 81671492), the Hunan Science and Technology Department (Grant No. 2018SK2102) and Hunan Education Department (Grant No. XJK011CGD001).

\section{Footnote}

Conflicts of Interest: The authors have no conflicts of interest to declare.

Ethical Statement: The authors are accountable for all aspects of the work in ensuring that questions related to the accuracy or integrity of any part of the work are appropriately investigated and resolved. Approval was given to the study by The Institutional Review Board (IRB) of Third Xiangya Hospital and Xiangya Hospital, Central South University. The procedure was performed in accordance with relevant guidelines and regulations. Informed consent was obtained after the procedure was fully explained from all participants and their legal guardians.

\section{References}

1. Asherman JG. Amenorrhoea traumatica (atretica). J Obstet Gynaecol Br Emp 1948;55:23-30.

2. Tan IF, Robertson M. The role of imaging in the investigation of Asherman's syndrome. Australas J Ultrasound Med 2011;14:15-8.

3. Foix A, Bruno RO, Davison T, et al. The pathology of postcurettage intrauterine adhesions. Am J Obstet Gynecol 1966;96:1027-33.

4. Salma U, Xue M, Md Sayed AS, et al. Efficacy of intrauterine device in the treatment of intrauterine adhesions. Biomed Res Int 2014;2014:589296.

5. Chen Y, Liu L, Luo Y, et al. Effects of Aspirin and Intrauterine Balloon on Endometrial Repair and Reproductive Prognosis in Patients with Severe Intrauterine Adhesion: A Prospective Cohort Study. Biomed Res Int 2017;2017:8526104.

6. Sugimoto O. Diagnostic and therapeutic hysteroscopy for traumatic intrauterine adhesions. Am J Obstet Gynecol 1978;131:539-47.

7. Gan L, Duan H, Xu Q, et al. Human amniotic mesenchymal stromal cell transplantation improves endometrial regeneration in rodent models of intrauterine adhesions. Cytotherapy 2017;19:603-16.

8. Hooker AB, Lemmers M, Thurkow AL, et al. Systematic review and meta-analysis of intrauterine adhesions after miscarriage: prevalence, risk factors and longterm reproductive outcome. Hum Reprod Update 2014;20:262-78.

9. Jones BP, Saso S, L'Heveder A, et al. The vaginal microbiome in uterine transplantation. BJOG 2020;127:230-8.

10. Kenyon C, Colebunders R, Crucitti T. The global epidemiology of bacterial vaginosis: a systematic review. Am J Obstet Gynecol 2013;209:505-23.

11. Ma H, Shi X, Fu Z, Wu X, Huangfu Y, Xu P, Zeng X, $\mathrm{Xu} \mathrm{J}$, Ruan H, Xu Q. Potential roles of microRNA in levonorgestrel-treated uterine leiomyoma cells. Transl 
Cancer Res 2018;7:1376-83.

12. Hay PE, Lamont RF, Taylor-Robinson D, et al. Abnormal bacterial colonisation of the genital tract and subsequent preterm delivery and late miscarriage. BMJ 1994;308:295-8.

13. Elovitz MA, Gajer P, Riis V, et al. Cervicovaginal microbiota and local immune response modulate the risk of spontaneous preterm delivery. Nat Commun 2019;10:1305.

14. Placek K, Schultze JL, Aschenbrenner AC. Epigenetic reprogramming of immune cells in injury, repair, and resolution. J Clin Invest 2019;129:2994-3005.

15. Lin XN, Zhou F, Wei ML, et al. Randomized, controlled trial comparing the efficacy of intrauterine balloon and intrauterine contraceptive device in the prevention of adhesion reformation after hysteroscopic adhesiolysis. Fertil Steril 2015;104:235-40.

16. Yu D, Wong YM, Cheong Y, et al. Asherman syndrome-one century later. Fertil Steril 2008;89:759-79.

17. Yu D, Li TC, Xia E, et al. Factors affecting reproductive outcome of hysteroscopic adhesiolysis for Asherman's syndrome. Fertil Steril 2008;89:715-22.

18. March CM. Asherman's syndrome. Semin Reprod Med 2011;29:83-94.

19. Laganà AS, Garzon S, Franchi M, Casarin J, Gullo G, Ghezzi F. Translational animal models for endometriosis research: a long and windy road. Ann Transl Med 2018;6:431.

20. Taskin O, Sadik S, Onoglu A, et al. Role of endometrial suppression on the frequency of intrauterine adhesions after resectoscopic surgery. J Am Assoc Gynecol Laparosc 2000;7:351-4.

21. Pabuccu R, Onalan G, Kaya C, et al. Efficiency and pregnancy outcome of serial intrauterine device-guided hysteroscopic adhesiolysis of intrauterine synechiae. Fertil Steril 2008;90:1973-7.

22. Deans R, Abbott J. Review of intrauterine adhesions. J Minim Invasive Gynecol;17:555-69.

23. Witkin SS, Linhares IM. Why do lactobacilli dominate the human vaginal microbiota? BJOG 2017;124:606-11.

24. Younes JA, Lievens E, Hummelen R, et al. Women and Their Microbes: The Unexpected Friendship. Trends Microbiol 2018;26:16-32.

25. Nasioudis D, Linhares IM, Ledger WJ, et al. Bacterial vaginosis: a critical analysis of current knowledge. BJOG 2017;124:61-9.

26. Moreno I, Codoñer FM, Vilella F, et al. Evidence that the endometrial microbiota has an effect on implantation success or failure. Am J Obstet Gynecol 2016;215:684-703.

27. Champer M, Wong AM, Champer J, et al. The role of the vaginal microbiome in gynaecological cancer. BJOG 2018;125:309-15.

28. Jenmalm MC. The mother-offspring dyad: microbial transmission, immune interactions and allergy development. J Intern Med 2017;282:484-95.

29. Blaser MJ, Dominguez-Bello MG. The Human Microbiome before Birth. Cell Host Microbe 2016;20:558-60.

30. Cash HL, Whitham CV, Behrendt CL, et al. Symbiotic bacteria direct expression of an intestinal bactericidal lectin. Science 2006;313:1126-30.

31. Dethlefsen L, McFall-Ngai M, Relman DA. An ecological and evolutionary perspective on human-microbe mutualism and disease. Nature 2007;449:811-8.

32. Ley RE, Peterson DA, Gordon JI. Ecological and evolutionary forces shaping microbial diversity in the human intestine. Cell 2006;124:837-48.

33. Ley RE, Turnbaugh PJ, Klein S, et al. Microbial ecology: human gut microbes associated with obesity. Nature 2006;444:1022-3.

34. Mazmanian SK, Liu CH, Tzianabos AO, et al. An immunomodulatory molecule of symbiotic bacteria directs maturation of the host immune system. Cell 2005;122:107-18.

35. Turnbaugh PJ, Ley RE, Mahowald MA, et al. An obesityassociated gut microbiome with increased capacity for energy harvest. Nature 2006;444:1027-31.

36. Peterson J, Garges S, Giovanni M, et al. The NIH Human Microbiome Project. Genome Res 2009;19:2317-23.

37. Donders GG, Bosmans E, Dekeersmaecker A, et al. Pathogenesis of abnormal vaginal bacterial flora. Am J Obstet Gynecol 2000;182:872-8.

38. Watts DH, Fazzari M, Fazarri M, et al. Effects of bacterial vaginosis and other genital infections on the natural history of human papillomavirus infection in HIV-1infected and high-risk HIV-1-uninfected women. J Infect Dis 2005; 191:1129-39.

39. Wiesenfeld HC, Hillier SL, Krohn MA, et al. Bacterial vaginosis is a strong predictor of Neisseria gonorrhoeae and Chlamydia trachomatis infection. Clin Infect Dis 2003;36:663-8.

40. Lai SK, Hida K, Shukair S, et al. Human immunodeficiency virus type 1 is trapped by acidic but not by neutralized human cervicovaginal mucus. J Virol 2009;83:11196-200.

41. Boskey ER, Telsch KM, Whaley KJ, et al. Acid 
production by vaginal flora in vitro is consistent with the rate and extent of vaginal acidification. Infect Immun 1999;67:5170-5.

42. Zhou X, Bent SJ, Schneider MG, et al. Characterization of vaginal microbial communities in adult healthy women using cultivation-independent methods. Microbiology (Reading, Engl) 2004;150:2565-73.

43. Ma B, Forney LJ, Ravel J. Vaginal microbiome: rethinking health and disease. Annu Rev Microbiol 2012;66:371-89.

44. Gieseck RL, Wilson MS, Wynn TA. Type 2 immunity in tissue repair and fibrosis. Nat Rev Immunol 2018;18:62-76.

45. Fielding CA, Jones GW, McLoughlin RM, et al. Interleukin-6 signaling drives fibrosis in unresolved inflammation. Immunity 2014;40:40-50.

46. O'Reilly S, Ciechomska M, Cant R, et al. Interleukin-6 (IL6) trans signaling drives a STAT3-dependent pathway that leads to hyperactive transforming growth factor- $\beta$ (TGF- $\beta$ ) signaling promoting SMAD3 activation and fibrosis via Gremlin protein. J Biol Chem 2014;289:9952-60.

47. Zhang W, Wang W, Yu H, et al. Interleukin 6 underlies angiotensin II-induced hypertension and chronic renal

Cite this article as: Zhao $\mathrm{X}$, Zhao Q, Zhu $\mathrm{X}$, Huang H, Wan X, Guo R, Zhao Y, Chen D, Xu D. Study on the correlation among dysbacteriosis, imbalance of cytokine and the formation of intrauterine adhesion. Ann Transl Med 2020;8(4):52. doi: 10.21037/atm.2019.11.124 damage. Hypertension 2012;59:136-44.

48. Le TT, Karmouty-Quintana H, Melicoff E, et al. Blockade of IL-6 Trans signaling attenuates pulmonary fibrosis. J Immunol 2014;193:3755-68.

49. Pedroza M, Schneider DJ, Karmouty-Quintana H, et al. Interleukin-6 contributes to inflammation and remodeling in a model of adenosine mediated lung injury. PLoS ONE 2011;6:e22667.

50. González GE, Rhaleb NE, D'Ambrosio MA, et al. Deletion of interleukin-6 prevents cardiac inflammation, fibrosis and dysfunction without affecting blood pressure in angiotensin II-high salt-induced hypertension. J Hypertens 2015;33:144-52.

51. Zhang Y, Wang JH, Zhang YY, et al. Deletion of interleukin-6 alleviated interstitial fibrosis in streptozotocin-induced diabetic cardiomyopathy of mice through affecting TGF $\beta 1$ and miR-29 pathways. Sci Rep 2016;6:23010.

52. Cui $W$, Zhang H, Liu ZL. Interleukin-6 receptor blockade suppresses subretinal fibrosis in a mouse model. Int J Ophthalmol 2014;7:194-7. 